International Journal of Engineering \&Technology, $7(3.12)(2018) 1205-1209$
International Journal of Engineering \& Technology
SPC
Website: www.sciencepubco.com/index.php/IJET
Research paper

\title{
Design and Implementation of CNC Milling Bot for Milled Circuit Board Fabrication
}

\author{
Rajesh Kannan Megalingam ${ }^{1 *}$, Shree Rajesh Raagul Vadivel ${ }^{2}$, Sreekumar S ${ }^{3}$, Swathi Sekhar ${ }^{4}$, \\ Thejus R Nair ${ }^{5}$, Midhun $\mathbf{R R}^{6}$ \\ 1,2,3,4,5,6 Department of Electronics and Communication Engineering, Amrita School of Engineering, Amritapuri, \\ Amrita Vishwa Vidyapeetham, Amrita University, India \\ *Corresponding author E-mail: ${ }^{1}$ rajeshkannan@ieee.org
}

\begin{abstract}
This research paper presents an alternative way to fabricate a Milled Circuit Board (MCB) with the help of a Computer Numerical Controlled (CNC) milling robot. Printed Circuit Boards (PCB) are one of the most widely used control modules in electrical and electronic industries. In this research paper we propose a computer controlled milling machine which can be used for quick fabrication of double layered PCBs called MCB where a user wants to test the circuit without the need to wait for the conventional PCB fabrication. The end effector of the CNC milling machine has the provision to move in 3 axes-x, $\mathrm{y}$ and $\mathrm{z}$ in accordance with the input received from the controller. DipTrace, an EDA/CAD software is used for creating the schematic diagram for the milled circuit boards. The design to be engraved on the copper clad board is uploaded to a software called CopperCam, where it can be converted to G-code files after making the necessary modifications. These G-code files are then uploaded to the CNC machine using the software Universal G-code Sender, thus enabling the microcontroller to direct the rotation of motors and coordinate the movements of the end effector so as to engrave the board design on to a copper clad.
\end{abstract}

Keywords: milling; CNC; PCB fabricating; Milled Circuit Boards.

\section{Introduction}

PCB or printed circuit boards are one among the most exploited discoveries in the field of electronics. They are an integral part of all the electronic gadgets being used today in all circuits from basic circuits (toys, music players etc.) to high end ones (super computers). PCBs are thin boards which have copper tracks carved on it which join at different nodes. At each node, there will be holes drilled and these can be used to fix all the components. There are surface mounted ICs and in which case nodes need not have holes. Different components are soldered onto the PCB to electronically connect them. As a result, it reduces the use of wires in a circuit which improves the overall appearance of it. PCBs are cost efficient and highly reliable.

In 2014, the revenue of PCB manufacturing in the U.S ranged at about $\$ 44$ billion. Thus, designing these boards is essential and needs to be done with high precision which is a time consuming and tedious process. This is where the importance of Computer Numerical Control (CNC) machine comes. Even though PCB fabrication can be done manually with precision, the human mind finds it difficult to operate for long hours with high concentration which in turn results to errors in the fabrication of the PCB which may result to the improper functioning of the circuit. Also with the development in technology, PCB's are to be smaller in terms of the size and more complex in terms of the circuitry. Therefore, the process of error free manual fabrication was almost next to impossible. With automation catching up at a very high pace, the PCB fabrication process is simplified, yet not so attractive for freelancers and hobbyists who want to try a simple circuit on a single PCB but has to go through tedious PCB fabrication process with prolonged time and money. This paper presents a CNC bot which makes it an easier and effortless process for users who need one or few PCBs without any delay which we call Milled Circuit Board (MCB). It also reduces the possibility of errors and enhances safety as manual labour is decreased to a great extent. As a 3D printer is becoming all pervasive in research labs and even in houses, the MCB fabrication using CNC bot could also follow the same way. The machine uses a CAD software called DipTrace to produce the schematic diagram and the CopperCam software is used to export the G-code. This gcode file is uploaded to the bot with the help of another software called Universal G-code Sender which effectively controls the movement of the bot. The extruder includes a milling tool which is able to move in $\mathrm{X}, \mathrm{Y}$ and $\mathrm{Z}$ axis and can cover a workspace of $10 * 10 \mathrm{~cm}$. The mechanical parts are designed using SolidWorks and printed using a 3-D printer.

\section{Motivation}

In today's world, technology is the ultimate solution to everyday problems and inconveniences faced by mankind. Thus the demand for resolving these difficulties through technology in the form of electronic devices is on the rise. All electronic devices used now are equipped with a PCB board considering the advantages of a printed circuit board such as, their efficiency in power delivery, compact design, and availability of cost efficient models. Time to testing is very important in any field, industry or research, student or freelancer. Most of the time a researcher or a freelancer would not want to wait for long time for simple two layer PCB fabrication. At the same tifme it's a tedious process to fabricate a PCB in- 
house at research labs or schools. A CNC based MCB would be a great boon in such situations for those people who would want to get the PCB done in an hour to few hours.

\section{Related Works}

Experiments have been done in the past and even more are ongoing in the fields related to CNCs and PCB fabrication using CNCs. Throughout the course of our research work many such ideas have been of significant help of which a few are cited below. In the article, PCB automation [1], experiences regarding the automation of PCB boards using CNC machines have been described with emphasis on the advantages and disadvantages of the process. In the paper Control Module for CNC PCB milling machine [2], the author describes the three sub modules which have been used for interfacing and coordinating the entire process of PCB milling. The paper titled G-code Optimization Algorithm [3] deals entirely with the optimization of G-codes, which is of significant help in converting the PCB designs into G-codes. Advanced discussions on PCB milling have been provided in papers such as Influence of the milling depth [4] and spindle motor speed controller [5], the former describes the effects of the milling depth on the PCB board and the latter describes the ways to adjust the speed of the end effector for precision milling. In addition to discussion of fabrication and design [6], we also analysed the assembling and testing of PCBs [7]. The paper Design and Implementation of PCB Using CNC [8], highlights the stability and accuracy of the machine using a path planning through a co-ordinate measurement method and [9-10] cost effective and small scale industrial implementations were also examined. Methods to compensate for the geometric and force errors were taken into consideration [11]. The possibility of controlling the speed of the stepper motor in accordance with the G-code was also considered [12]. In the end, we studied the three different CNC controllers used in industries on the basis of parameters used [13]. Research papers [14 - 15] discuss about the implementation of a drawing bot and $3 \mathrm{D}$ printing bot similar to our proposed one.

\section{System Description}

System Architecture comprises of ten blocks. The design of the PCB to be engraved is drawn via Diptrace and uploaded to Coppercam software which converts it into G-code values. These values are then uploaded into Arduino Mega 2560 through CNC Shield. The respective co-ordinates of the G-code is then sent to the corresponding motor driver via Arduino Mega. The particular motor driver activates the motor which then provides linear motion to the pulley belt system. The required power is supplied by a $12 \mathrm{~V}$ lithium polymer battery. Limit switches are installed to make sure the machine works within the workspace. A DC power supply is directly given to the end effector.

The controlling part of the CNC milling machine includes the micro-controller Arduino UNO mounted on top with the CNC shield, which provides an interface between the micro-controller and the motor drivers. CNC shield holds the provision to connect up to 4 motor drivers of which two have been used for the two stepper motors for the $\mathrm{x}$ axis and one each for the $\mathrm{y}$ axis and $\mathrm{z}$ axis stepper motors. Stepper motor used is NEMA17 which is controlled using HR4998 stepper motor drivers fixed on the CNC shield. The effect of rotation of each stepper motors is delivered to the linear slides using the GT2 timing belts attached to the linear slides, two fixed on the x-axis and one on the y-axis. Limit switches are used to prevent sudden hike in current drawn by the stepper motors if the slides ever hit the metal rods at the end. End effector used is Dremel.

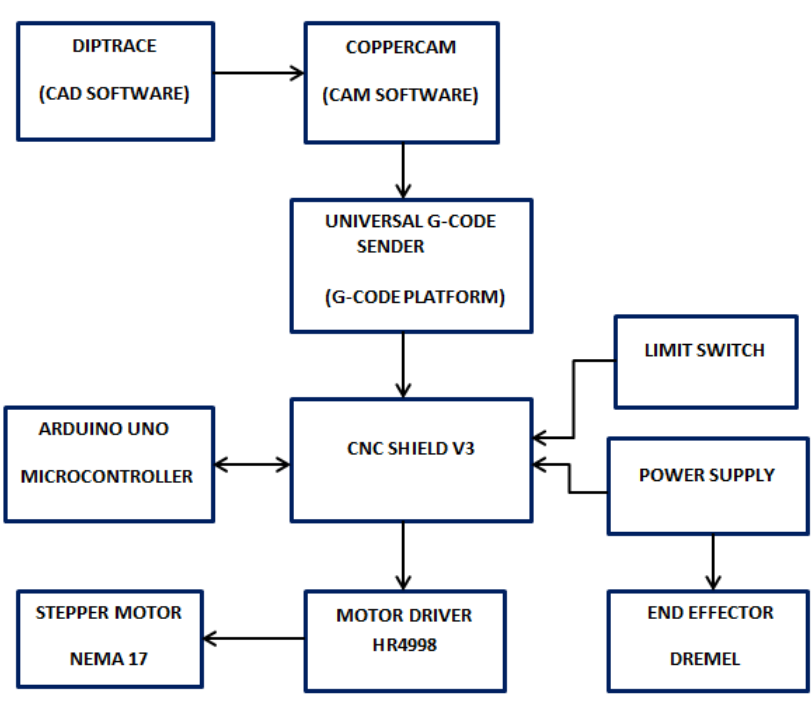

Fig. 1: System Architecture

\section{Design and Implementation}

\subsection{Design}

The entire structure of the CNC milling bot is designed and developed using SolidWorks. Apart from the hardware components mentioned above most of the parts used in this project are designed in house and 3D printed. This ensured our demand for custom and specific parts for a specific purpose and that our entire cost for this design and manufacturing remained low. Fig. 5 shows the entire structure of our milling bot design in SolidWorks. The extreme base of the structure is a square shape with one metal rod on each side, one pair of opposite sides of this square structure bears linear slides as shown in Fig. 5.

At the end of the metal rods bearing a linear slide is a motor and pulley, each fixed at the extreme ends using motor holder as shown in Fig. 2a and pulley holder as in Fig. 2b. A GT2 timing belt connects the motor and the pulley as in Fig. $2 \mathrm{c}$ to the linear slides. This ensures the power developed by the motor is contributed to the motion of the linear slides without significant loss. A 3D printed part is used to connect the timing belt to the linear slides as in Fig. 3a. These two slides mentioned above contribute to the $\mathrm{y}$-axis motion of the CNC machine. A different rod is placed above the two linear slides connecting them, another 3D printed part is placed along this rod which replaces the linear slides mentioned above. This is given in Fig. 3b, which contributes to the $\mathrm{x}$-axis movement. The compartment bearing the end effector holder as shown in Fig. 3c and the mechanism for motion along the $\mathrm{z}$-axis direction is attached to the $\mathrm{x}$-axis slider. Gear nut mechanism is used for motion of the end effector holder along the $\mathrm{z}$-axis direction. Figure $4 \mathrm{a}$ and $4 \mathrm{~b}$ shows the $\mathrm{z}$-axis motion part and rob support design used in the building of the milling bot.

Parts designed in SolidWorks:

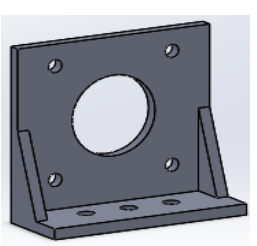

(a)

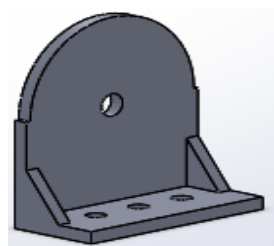

(b)

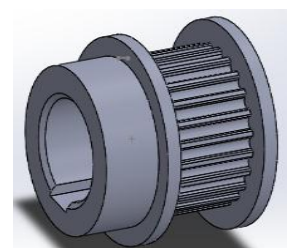

(c)
Fig. 2: (a) Motor holder, (b) Pulley holder, (c) Pulley 


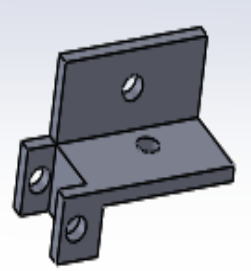

(a)

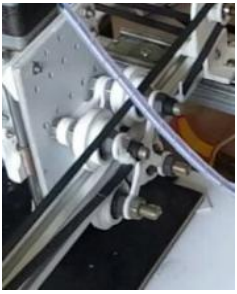

(b)

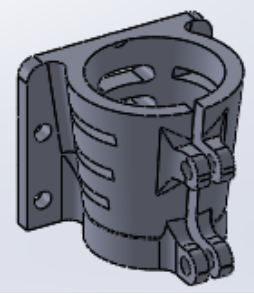

(c)
Fig. 3: (a) Slide to belt connector, (b) Roller, (c) Holder

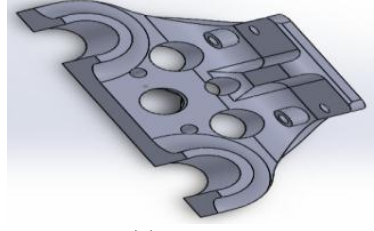

(a)

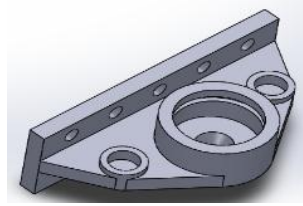

(b)
Fig. 4: (a) Z-axis motion part, (b) Rod support

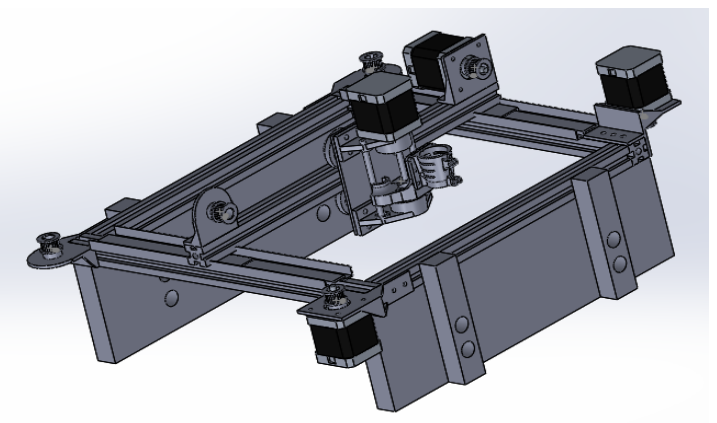

Fig. 5: SolidWorks Design of Milling Bot

\subsection{Implementation}

Working of the machine is controlled by the motor driver, HR4998. The G-code values are passed onto the Arduino mega upgraded with a CNC shield. The stepper motors are attached to pulleys on the other side using belts. Also, a slider is coupled to the metal rod for easy and friction free movement. This is done on two sides of the square. The ends of a metal rod are connected to these sliders. When the stepper motor rotates, it leads to the linear movement of the slider and the metal rod. This constitutes the movement in the y-axis. Next the stepper motor-pulley attachment is modeled on this rod and sliders and installed on both top and bottom of it which results in $\mathrm{x}$-axis movement.

The entire setup in which the parts fabricated using the 3D printer and assembled with other components and materials are shown in the image in Fig 6.The z-axis motion is controlled by a motor which is connected to the gear nut mechanism. The rotation of the motor controls the vertical movement of this holder. The end effector is placed in the end effector holder and switched on.

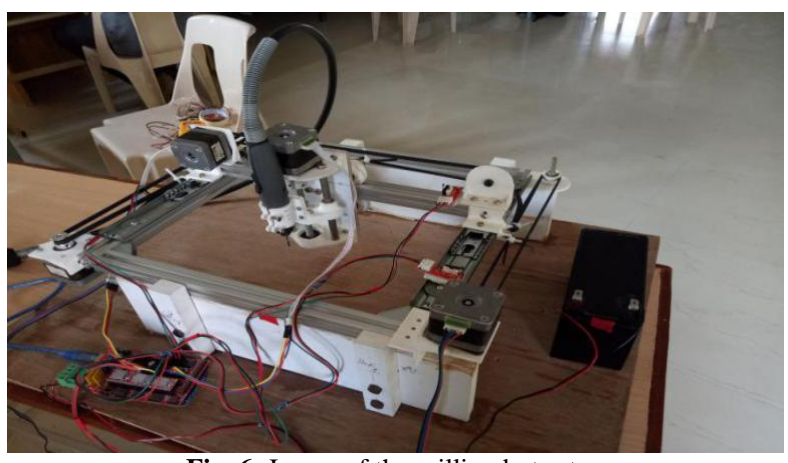

Fig. 6: Image of the milling bot setup

\subsection{Determining the Home Position}

The position of the $\mathrm{x}$-axis, $\mathrm{y}$-axis and $\mathrm{z}$-axis of the $\mathrm{CNC}$ machine when it is connected to the system will be assumed as the home position of the $\mathrm{x}$-axis, $\mathrm{y}$-axis and $\mathrm{z}$-axis respectively for any design uploaded to the CNC machine throughout the time this connection remains.

\section{Software Integration}

The proper functioning of a $\mathrm{CNC}$ milling machine is a three step process. First, a CAD software is required for designing the PCB. Second, a CAM software for converting these into G-codes and finally a CNC control software which feeds the g-codes to bring the machine to life. The CAD software used is DipTrace a sample of which is shown in Fig. 7 which is a design software specifically for PCBs. Here, the most elaborate and complex circuits can easily be designed.

The designed circuit is fed into the CAM software, CopperCAM which is shown in Fig. 8, which converts the design into specific G-codes for engraving, drilling and cutting. It also enables us to modify the milling procedure as in the size of the holes and threads, depth of drilling and also the speed of the spindle and depending upon our specifications, G-codes are generated. Gcodes are numerical control codes which consist of the different coordinate values at every distinct position of the design. Once gcodes are generated, this is to be fed into the micro-controller. The software we used for this purpose is Universal G-code sender as in Fig.9, which is a Java application for interfacing with the CNC shield. The function of a CNC shield is to convert G-codes into stepper signals. The orchestrated rotation of the motors results in the movement of the belt which directs the end effector to the desired position. In addition to this, another software used is Arduino IDE to which the GRBL library is added and this is uploaded onto the Arduino Uno. The simulation of the PCB milling process can be obtained using the Mach3 software as shown in Fig. 10 .

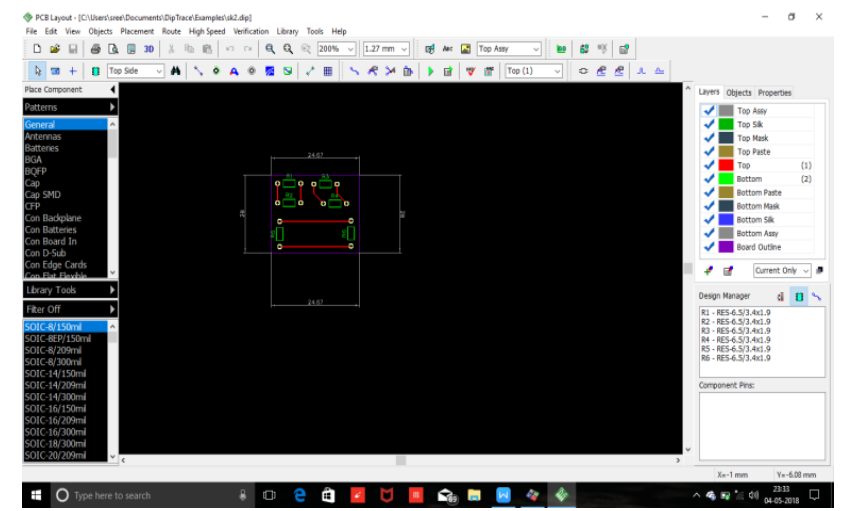

Fig. 7: DipTrace

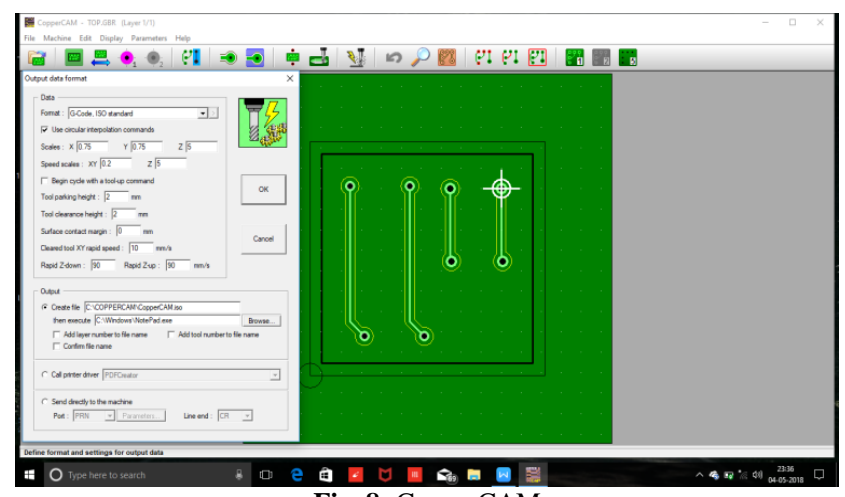

Fig. 8: CopperCAM 


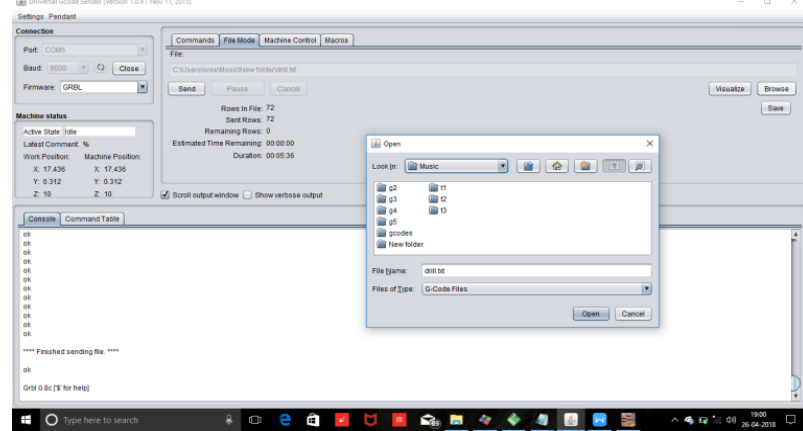

Fig. 9: Universal G-code Sender

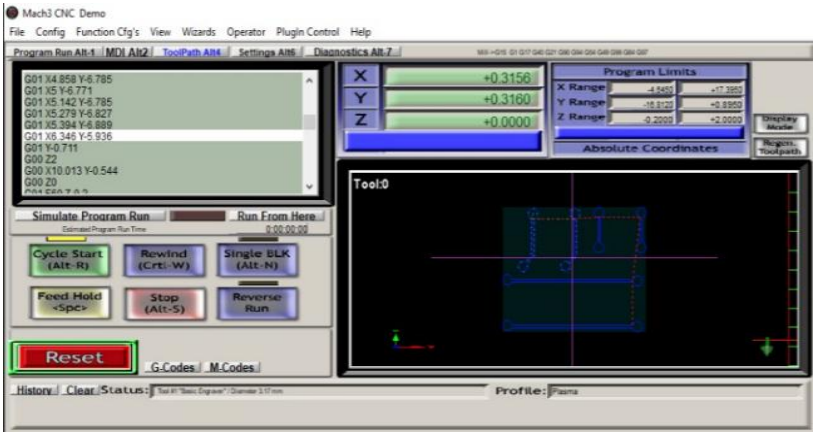

Fig. 10: Simulation on Mach3

\section{Experiments and Results}

Different designs as given in Fig. 11 with varying complexity were drawn in DipTrace software and uploaded to copperCAM. CopperCAM software helps to convert these designs into Gcodes values which can be directly interfaced with the PCB milling machine. CopperCAM is again used to define the milling tool dimensions used for engraving and drilling process. The output parameters like speed of the $\mathrm{x}$-axis, $\mathrm{y}$-axis and z-axis motors, scale ratios on each axis and other parameters like tool clearance height etc can be defined.These values are very significant as these directly affect the speed of the milling process and the dimensions of the design being drawn.CopperCAM develops to Gcode files, one for engraving process and another one for the drilling process. Gcode file for engraving is first uploaded to the $\mathrm{CNC}$ machine using the Universal Gcode Sender software, after the engraving process is over the Gcode file for drilling process is uploaded. It is very important that the position of the copper clad boards should not be changed after the engraving process, as it could result in the dis positioning of holes on the board.

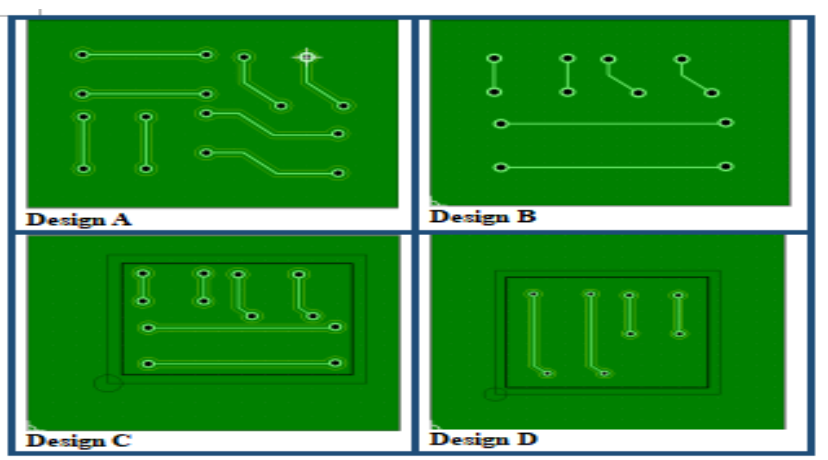

Fig. 11: Four different Designs

The tests were carried out on one sided copper clad boards of dimension $15 * 10 \mathrm{~cm}$.These dimensions were chosen because our work area was bound to $10 * 10 \mathrm{~cm}$. The milling tool dimension was set to $1 \mathrm{~mm}$ and a $1 \mathrm{~mm}$ milling tool was used throughout the process. The engraved design of Design $\mathrm{C}$ on a copper clad board is shown in Fig. 12.
From Table1, we see that the total time taken is high for Design A compared to the rest, whereas time is the least for Design D.

Order of engraving time:

Design A > Design C > Design B > Design D

Order of Drilling Time:

Design A > Design C > Design B > Design D

Hence, the Total Time taken is a combination of the Engraving Time and Drilling Time.

Time Taken is proportional to Drilling Time and Engraving Time. Increasing order of duration to complete the work is as follows:

Design $\mathrm{D}<$ Design $\mathrm{B}<$ Design $\mathrm{C}<$ Design A

Table 1: MCB Timings

\begin{tabular}{|l|l|l|l|l|}
\hline Time/Design & Design A & Design B & Design C & Design D \\
\hline Engraving Time & $1 \mathrm{~m}: 21 \mathrm{~s}$ & $0 \mathrm{~m}: 57 \mathrm{~s}$ & $1 \mathrm{~m}: 02 \mathrm{~s}$ & $0 \mathrm{~m}: 42 \mathrm{~s}$ \\
\hline Drilling Time & $5 \mathrm{~m}: 36 \mathrm{~s}$ & $4 \mathrm{~m}: 08 \mathrm{~s}$ & $4 \mathrm{~m}: 35 \mathrm{~s}$ & $2 \mathrm{~m}: 48 \mathrm{~s}$ \\
\hline Total Time & $6 \mathrm{~m}: 57 \mathrm{~s}$ & $5 \mathrm{~m}: 05 \mathrm{~s}$ & $5 \mathrm{~m}: 37 \mathrm{~s}$ & $3 \mathrm{~m}: 30 \mathrm{~s}$ \\
\hline
\end{tabular}

As the complexity of the design increases, the time taken for PCB fabrication also increases. The time taken for drilling process is more compared to time taken for engraving process. It can be observed from the table that there is a high influence of drilling time on the total time taken for the PCB fabrication, if the time consumed for drilling process can be reduced, the entire PCB fabrication process can be made faster.

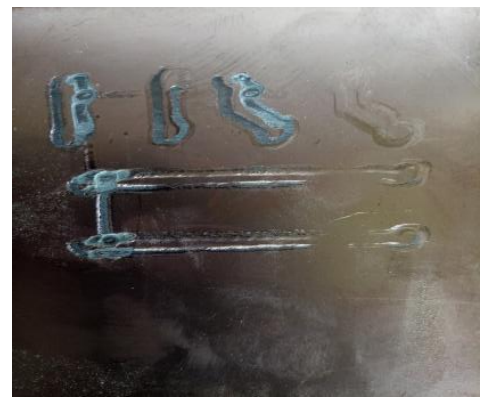

Fig. 12: Sample output

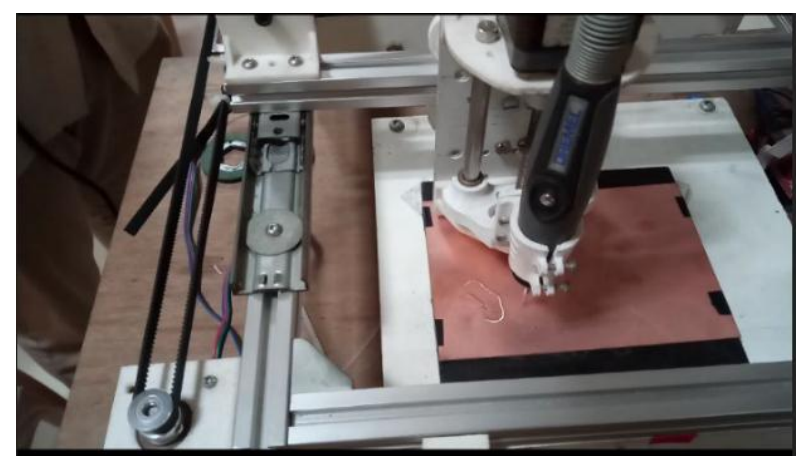

Fig. 13: CNC Milling Process

\section{Conclusion and Future Works}

This project primarily aims at making the process of PCB fabrication a more easier and accurate process. We also aim to incorporate the performance and efficiency of an industrial milling machine into a compact, cost efficient version. The machine was constructed with parts designed using SolidWorks to further emphasise the latter statement. A CNC shield is mounted on the Arduino Uno for improving the features and power capacity. Sliders are installed on the axes for the ease of movement. The UGS software when provided with proper G-code ensures the proper movement of the stepper motors. Limit switches ensures that the robot works within the workspace. Various designs were given as input, and the time for completing the task were recorded as a sum of engraving and drilling time. One of the inferences was that drilling time took a longer duration than engraving time. Also time to complete the process was dependent on complexity of the de- 
sign. The results were promising which shows it does not compromise in terms of performance or efficiency.

For future objectives, our main objective is to increase our work area. By doing so we will be able to manufacture PCBs of larger dimensions. Furthermore, we aim to increase the speed in which the extruder fabricates. To achieve this, development of a better code as well as use of more powerful motors are required. In addition to this, we also aim to explore better technologies and innovative ideas like an IoT interface that can be implemented in this robot.

\section{Acknowledgement}

We would like to thank the Almighty for giving us an opportunity to make this paper a success. We would also like to show our gratitude towards Amrita University and the Humanitarian Technology, HuT Labs for their expert guidance throughout this project. We would also like to thank our family and friends for their constant support and encouragement.

\section{References}

[1] Ricardo Salvador Guadrón Gutiérrez; Juan José Guevara Vásquez, "Experiences in automated construction of PC boards using an open source cnc machine", 2015 IEEE Thirty Fifth Central American and Panama Convention (CONCAPAN XXXV).

[2] Ariana Tulus Purnomo; Farkhad Ihsan Hariadi; Arif Sasongko, "Development of interface and coordination for control module CNC PCB milling machine", 2015 International Symposium on Intelligent Signal Processing and Communication Systems (ISPACS).

[3] Razvan-Mihai Aciu; Horia Ciocarlie, "G-code optimization algorithm and its application on Printed Circuit Board drilling”, 2014 IEEE 9th IEEE International Symposium on Applied Computational Intelligence and Informatics (SACI).

[4] M. Gatchev; S. Kamenopolsky; V. Bojanov; P. Dankov, "Influence of the milling depth on the microstrip parameters in milled PCBplates for microwave applications", 14th International Conference on Microwaves, Radar and Wireless Communications. MIKON 2002. Conference Proceedings (IEEE Cat.No.02EX562).

[5] Fiqih Tri Fathulah Rusfa; Farkhad Ihsan Hariadi; Arif Sasongko, "Development of an FPGA-based sub-module as three-phase spindle motor speed controller for CNC PCB milling machine", 2015 International Conference on Electrical Engineering and Informatics (ICEEI).

[6] Christopher F. Wise," Fabrication of Printed Circuit Boards Using a Table Top CNC Mill”, the Technology Interface/Fall 2007

[7] Khandpur, R. S. (2005). Printed Circuit Boards: Design, Fabrication, Assembly and Testing. Tata McGraw-Hill Education. ISBN 9780070588141.

[8] Prabhanjay Gadhe, Vikas Jangir, Mayur Yede, Wasim-Ul-Haq, "Design and Implementation of PCB Using CNC", International Research Journal of Engineering and Technology (IRJET) Volume: 04 Issue: 02,Feb -2017

[9] Motaz Daadoo, Yousef-Awwad Daraghmi, "Design and Implementation of Low Cost Computer Numerical Control-Printed Circuit Boards Drilling Machine”, International Journal of Engineering and Innovative Technology (IJEIT) Volume 5, Issue 10, April 2016

[10] H. Ferdinando, I. N. Sandjaja, and G. Sanjaya, "Automatic Drilling Machine for Printed Circuit Board," Proceedings of The 6th Symposium on Advanced Intelligent Systems, Surabaya Indonesia., pp. 218-222, September 2005

[11] Chana Raksiri Manukid Parnichkun, "Geometric and force errors compensation in a 3-axis CNC milling machine", International Journal of Machine Tools and Manufacture Volume 44, Issues 12 13, October 2004

[12] Yin He, Wen Quangang, Lin Gang, "Research on the control method of 3D printer based on FDM technology", Mechanical and Intelligent Manufacturing Technologies (ICMIMT), International Conference, Cape Town, South Africa

[13] Rajendra Rajput, Dr. Ajay Kumar Sarathe, "Comparative Study of CNC Controllers used in CNC Milling Machine", American Journal of Engineering Research (AJER) e-ISSN: 2320-0847 p-ISSN : 2320-0936 Volume-5, Issue-4, pp-54-62
[14] Megalingam, R.K., Raagul, S., Dileep, S., Sathi, S.R., Pula, B.T., Vishnu, S.r, Sasikumar, V., Gupta, U.S.C., "Design, implementation and analysis of a low cost drawing bot for educational purpose", International Journal of Pure and Applied Mathematics, Volume 118, Issue 16 Special Issue, 2018, Pages 213-230

[15] Rajesh Kannan Megalingam, Shreerajesh Raagul, Sarveswara Reddy Sathi, Bhanu Teja Pula, Souraj Vishnu P, Vishnu Sasikumar, Uppala Sai Chaitanya Gupta, "Cost-effective, Custom-made, 3D Printer Design and Fabrication for Educational Purposes, 2018 International Conference on Trends in Electronics and Informatics (ICOEI 2018) 